\title{
Lernförderliche Arbeitssysteme für die Arbeitswelt von morgen
}

\author{
Roman Senderek
}

\subsection{Wandel der Arbeitswelt}

Die Digitalisierung und Vernetzung von Produktions- und Dienstleistungsprozessen bieten erhebliche wirtschaftliche Potenziale für Unternehmen. Schätzungen gehen davon aus, dass die Digitalisierung mit etwa $1 \%$ zum Wirtschaftswachstum in Deutschland beitragen wird (Rüßmann et al. 2015, S. 8). Weitere Prognosen sehen bis zum Jahr 2025 eine kumulierte Produktivitätssteigerung von $30 \%$ mit einer möglichen Bruttowertschöpfung von rd. 80 Milliarden Euro (BITKOM und Fraunhofer IAO 2014, S. 6). Auch wenn die digitalisierte und vernetzte Produktion im Sinne der Industrie 4.0 heute noch nicht in der Breite bei deutschen Unternehmen umgesetzt ist, sind bereits erste Ansätze und Konzepte in unterschiedlichen Branchen erkennbar (Lukas et al. 2014, S. 256). So geben laut einer Studie bereits $17 \%$ der befragten deutschen Unternehmen an, über dezentral vernetzte, selbststeuernde Produktionsprozesse zu verfügen (Pierre Audoin Consultants GmbH 2014, S. 10). Der industrielle Wandel wird auch daran deutlich, dass die Relevanz von „Industrie 4.0“ in den vergangenen Jahren für Unternehmen stark zugenommen hat (Pierre Audoin Consultants GmbH 2014, S. 10). Grundsätzlich wird auch deutlich, dass sich der Implementierungsgrad in Abhängigkeit der jeweiligen Branche sichtbar voneinander unterscheidet.

Insbesondere der Automotive-Sektor gilt bei der Implementierung von „Industrie 4.0“-Konzepten als führend. Das durch „Industrie 4.0“ in den kommenden zehn Jahren zusätzlich erreichbare Wertschöpfungspotenzial wird auf 15 Milliarden Euro bei einer

R. Senderek $(\square)$

FIR e. V. an der RWTH Aachen, Institute for Industrial Management at RWTH Aachen University, Campus-Boulevard 55, 52074 Aachen, Deutschland

e-mail: Roman.Senderek@fir.rwth-aachen.de 
jährlichen Wachstumsrate von 1,5 \% geschätzt (BITKOM und Fraunhofer IAO 2014, S. 7). Gleichzeitig weist die Automotive-Industrie auch einen der höchsten Automatisierungsgrade auf. Für einen Zeithorizont von fünf Jahren wird in der Automobil- und Fertigungsindustrie eine weitere deutliche Zunahme der Automatisierung und insbesondere ein deutlicher Trend von der teilautomatisierten hin zu einer vorwiegend oder voll automatisierten Produktion prognostiziert (Kelkar et al. 2014, S. 45). Dem steht im Rahmen dieser Prognose allerdings ein relativ moderates Absinken der vorwiegend manuellen oder rein manuellen Produktion von $26 \%$ auf $18 \%$ gegenüber (Kelkar et al. 2014, S. 45). Dies könnte damit zu erklären sein, dass der Anteil manueller Routinearbeit bereits heute nur noch einen geringen Anteil ausmacht.

Die Digitalisierung von Produktions- und Dienstleistungsprozessen geht auch mit fundamentalen Veränderungen der Arbeitswelt einher. Dabei wird sich die Rolle des Menschen in der industriellen Produktion erheblich wandeln. Durch die zunehmend komplexer werdenden, echtzeitgesteuerten Arbeits- und Produktionssysteme werden sich die Arbeitsinhalte und -prozesse, aber auch die Anforderungen an Fähigkeiten und Kompetenzen der Beschäftigten verändern (Kagermann et al. 2013, S. 6). Dies wird sich deutlich auf den Qualifizierungsbedarf sowie die Notwendigkeit der Kompetenzentwicklung auswirken. Inwiefern sich Tätigkeiten und Aufgaben verändern werden, kann derzeit allerdings nur anhand begrenzter verfügbarer Studien abgeschätzt werden. So gaben $80 \%$ der befragten Unternehmen einer Studie an, dass die zunehmend flexible Produktionsarbeit eine zusätzliche Qualifizierung ihrer Mitarbeiter erfordere (Spath et al. 2013, S. 86). Unternehmen sind daher gefordert, neue Konzepte und Strategien für die innerbetriebliche Qualifizierung und Personalentwicklung zu erarbeiten. Es gilt Arbeits- und Produktionssysteme so zu planen und zu gestalten, dass sie arbeitsintegrierte sowie bedarfsgesteuerte Formen des Lernens unterstützen (Kagermann et al. 2013, S. 59 ff.). Auch hierbei können die technologischen Entwicklungen der letzten Jahre einen bedeutenden Beitrag leisten, die Beschäftigten für ihre zukünftigen Aufgaben zu befähigen. Diese Potenziale müssen jedoch erkannt und zielgerichtet ausgeschöpft werden (Müller 2015, S. 1). Es gilt aber in gleichem Maße auch eine Reihe von Herausforderungen zu meistern. Fragen zu ITSicherheit, Datenschutz und Arbeitsorganisation müssen dabei ebenso frühzeitig anvisiert werden wie die Entwicklung einheitlicher Normen und Standards.

Die Prognosen zu Auswirkungen auf die Beschäftigung in Deutschland sind bisher eher uneindeutig. Während beispielsweise die Beratungsgesellschaft BCG einen Verlust von 600.000 Arbeitsplätzen bei allerdings 950.000 neuen Arbeitsplätzen in Deutschland in den kommenden Jahren sieht, geht der Branchenverband BITKOM im Bereich des Maschinenbaus von bereits entstandenen 28.000 zusätzlichen Arbeitsplätze aus (BITKOM 2014). Daneben gibt es auch Prognosen, die von deutlich negativeren Effekten ausgehen, wie z. B. die vielzitierte Studie von Frey und Osborne (Frey und Osborne 2013) oder ein in der Wirtschaftswoche (Tutt 2015) veröffentlichter Beitrag. Die Betrachtung von konkreten Fallbeispielen kann hier jedoch einen ersten Aufschluss über die Veränderungen der Arbeitswelt geben. 
Vor diesem Hintergrund werden im Rahmen des durch das Bundesministerium für Bildung und Forschung (BMBF) geförderten und vom Projektträger im DLR-begleiteten Verbundprojekt ELIAS neue Ansätze für die Gestaltung von Arbeits- und Produktionssystemen entwickelt. Das Akronym ELIAS steht für Engineering und Mainstreaming lernförderlicher industrieller Arbeitssysteme für die Industrie 4.0. Die Verbundpartner FIR e. V. an der RWTH Aachen, XERVON Instandhaltung GmbH, Deutsche MTM-Vereinigung e. V., Werkzeugmaschinenlabor (WZL) der RWTH Aachen, Zwiesel Kristallglas AG, HELLA KGaA Hueck \& Co. sowie FEV GmbH verfolgen dabei gemeinsam das Ziel, ein Konzept zu erarbeiten, welches das Lernen im Prozess der Arbeit als elementaren gestalterischen Bestandteil in bestehende oder zukünftige Arbeitssysteme integriert. Das angestrebte Ergebnis ist ein Planungsinstrument, dessen Funktionsweise in Abb. 7.1 dargestellt ist.

Bei der Auswahl von arbeitsorientierten oder technologiegestützten Lernformen sind die jeweiligen Rahmenbedingungen eines Unternehmens zu untersuchen. $\mathrm{Zu}$ diesen zählen die Arbeitsaufgaben und der damit verbundene Lerngehalt, die technologisch-mediale Infrastruktur sowie der Lerntyp des Unternehmens. Zudem ist die installierte Basis an Lernlösungen und Lerninhalten zu berücksichtigen, die im Unternehmen derzeit eingesetzt wird. Auf Basis dieser Analyse der Rahmenbedingungen ermöglicht das Planungsinstrument dienstleistenden und produzierenden Unternehmen eine sinnvolle Auswahl von Lernlösungen, um Lernförderlichkeit in ihre zukünftigen, aber auch derzeitigen Arbeitsund Produktionssysteme zu integrieren.
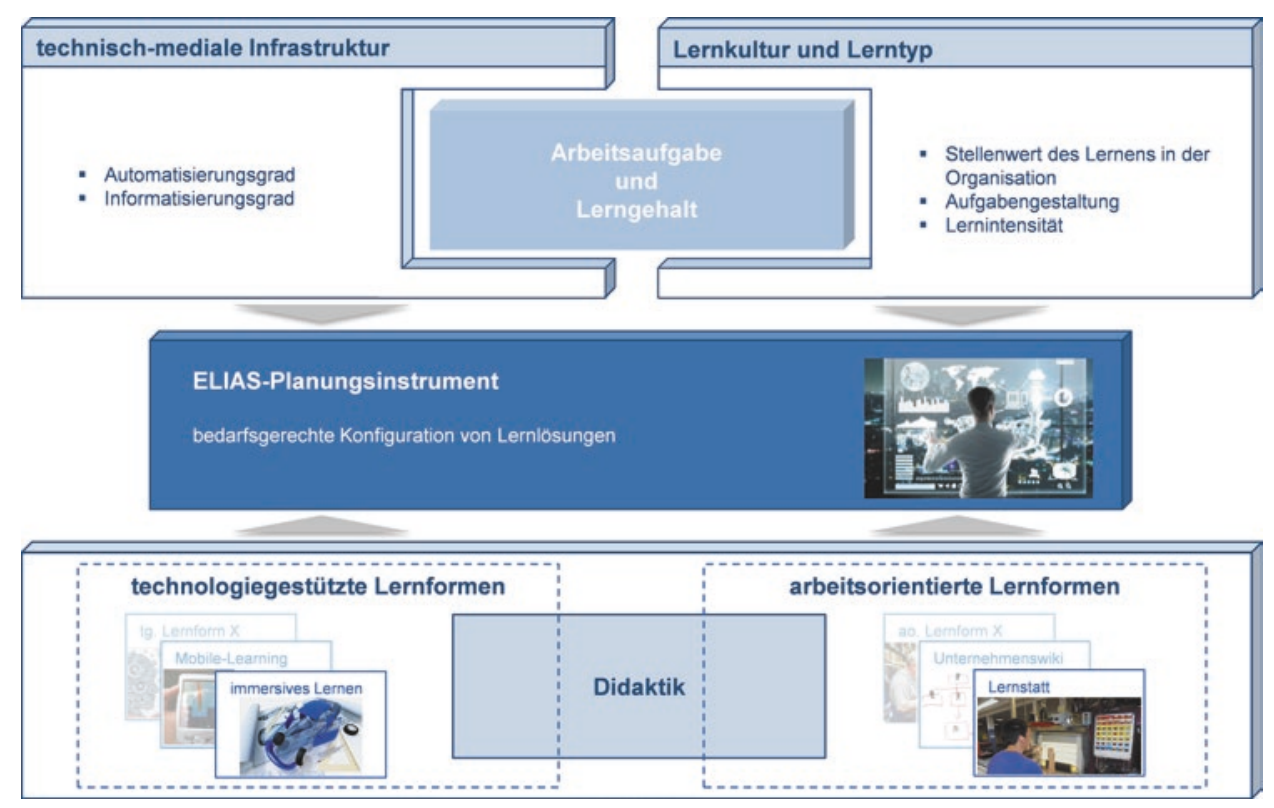

Abb. 7.1 Funktionsweise des ELIAS-Planungsinstruments 


\subsection{ELIAS in der Praxis: Kurzvorstellung der Unternehmen}

Die industrielle Anwendbarkeit des ELIAS-Ansatzes wird mithilfe der Use-Cases der Industriepartner sowie der Entwicklung von Demonstratoren erprobt und weiterentwickelt. Beispielhaft werden in diesem Beitrag für den Produktionssektor das Arbeitssystem der HELLA KGaA Hueck \& Co. sowie für den Bereich der Dienstleistungserstellung das Arbeitssystem der FEV GmbH detailliert analysiert.

Die HELLA KGaA Hueck \& Co. ist ein international operierender Automobilzulieferer mit Hauptsitz in Lippstadt. Das Unternehmen wurde 1899 unter dem Namen „Westfälische Metall-Industrie Aktien-Gesellschaft (WMI)“ gegründet. Heute zählt der HELLA Konzern zu den 40 weltweit führenden Automobilzulieferern sowie zu den 50 größten deutschen Industrieunternehmen. Weltweit sind etwa 32.000 Menschen an mehr als 100 Standorten in über 35 Ländern beschäftigt. Das Kerngeschäft des Unternehmens gliedert sich in die Segmente Automotive, Aftermarket und Special Applications, dargestellt in Abb. 7.2.

Der HELLA Konzern ist auf innovative Lichtsysteme und Fahrzeugelektronik spezialisiert. Im Geschäftsbereich Licht entwickelt und fertigt HELLA verschiedene Scheinwerfer, Leuchten und Innenbeleuchtung. Das Segment der Fahrzeug-Elektronik umfasst Systeme zur Effizienzsteigerung sowie Sicherheits- und Komfortsysteme. Im Bereich Aftermarket verfügt das Unternehmen über eine der größten Handelsorganisationen für Kfz-Teile, -Zubehör, Diagnose und Serviceleistungen in Europa. Produkte für Spezialfahrzeuge und fahrzeugunabhängige Anwendungen, wie Straßen- oder Industriebeleuchtung werden im Special Applications-Segment entwickelt, gefertigt und vertrieben.

Die FEV GmbH (Forschungsgesellschaft für Energietechnik und Verbrennungsmotoren) ist ein international tätiges Dienstleistungsunternehmen in der Konstruktion und Entwicklung von Antrieben. Gegründet 1978, hat die FEV ihren Hauptsitz in Aachen. Derzeit sind im Unternehmen etwa 4000 Mitarbeiter in 35 Standorten auf vier Kontinenten beschäftigt. Heute reicht das Leistungsspektrum weit über das ursprüngliche Hauptbetätigungsfeld hinaus und ist in Abb. 7.3 dargestellt.

\begin{tabular}{|c|c|c|c|c|c|c|}
\hline \multicolumn{7}{|c|}{ Geschäftssegmente } \\
\hline \multicolumn{2}{|c|}{ Automotive } & \multicolumn{3}{|c|}{ Aftermarket } & \multicolumn{2}{|c|}{ Special Applications } \\
\hline Licht & Elektronik & Han & del u. Werkstät & ten & $\begin{array}{c}\text { spezielle } \\
\text { Erstausrüstung }\end{array}$ & Industries \\
\hline
\end{tabular}

Abb. 7.2 Kerngeschäfte der HELLA KGaA Hueck \& Co. 


\begin{tabular}{|c|c|c|c|c|}
\hline \multicolumn{5}{|c|}{ Geschäftsbereiche } \\
\hline \multicolumn{2}{|c|}{ Engineering Dienstleistungen } & \multicolumn{2}{|c|}{ Test Systeme } & \multirow[b]{2}{*}{\begin{tabular}{l}
\multicolumn{1}{c}{ Software } \\
- Prüf- und Messtechnik- \\
systeme \\
- Entwicklung eingebetteter \\
Systeme \\
- Datenmanagement u. \\
Analyse \\
- modellbasierte \\
Kalibrierung \\
- virtual Engine- \\
Dynamiksimulation für \\
den Antriebsstrang
\end{tabular}} \\
\hline $\begin{array}{l}\text { Personenwagen- } \\
\text { und leichte } \\
\text { Nutzfahrzeug- } \\
\text { Dieselmotoren } \\
\text { - PKW- } \\
\text { Ottomotoren } \\
\text { - Nutzfahrzeug- } \\
\text { und Industrie- } \\
\text { motoren } \\
\text { - Großmotoren } \\
\text { - Getriebe } \\
\text { - Turbo-Aufladung }\end{array}$ & $\begin{array}{l}\text { - Fahrzeugtechnik } \\
\text { - Fahrwerkskonstruktion } \\
\text { - Bordnetz und Elektronik } \\
\text { - Hybridfahrzeuge und E- } \\
\text { Mobility } \\
\text { - Fahrzeugelektronik, } \\
\text { Infotainment und Telematik } \\
\text { - NVH - Noise Vibration } \\
\text { Harshness } \\
\text { - High Tech-Anwendungen } \\
\text { - Produktionstechnik und } \\
\text { Value Engineering }\end{array}$ & $\begin{array}{l}\text { Service } \\
\text { - Beratung, } \\
\text { Konzeptentwicklung } \\
\text { u. Planung } \\
\text { - Programm- } \\
\text { Engineering } \\
\text { - schlüsselfertige } \\
\text { Systemintegration } \\
\text { - Installation u. } \\
\text { Kommissionierung } \\
\text { - Service u. Wartung }\end{array}$ & $\begin{array}{l}\text { Produkte } \\
\text { - Datensysteme } \\
\text { - Konditionierungs- } \\
\text { einheiten } \\
\text { - Messinstrumente } \\
\text { - Dynamometer } \\
\text { - Sonderprü- } \\
\text { stande } \\
\text { - Software }\end{array}$ & \\
\hline
\end{tabular}

Abb. 7.3 Geschäftsbereiche der FEV GmbH

Im Bereich der Engineering-Dienstleistungen bietet das Unternehmen seinen Kunden die Entwicklung, Konstruktion und das Prototyping von Verbrennungsmotoren sowie Getrieben in den Bereichen Ottomotoren, Dieselmotoren und Nutzfahrzeugmotoren. Weitere Bereiche, in denen die FEV heute tätig ist, sind Elektrik/Elektronik, innovative Fahrzeugkonzepte, aber auch elektrifizierte oder hybride Antriebe und alternative Kraftstoffe. Zudem hat die FEV ihr technisches Portfolio auf andere industrielle Sektoren ausgeweitet, wie bspw. die Transportbereiche Marine, Schiene und Luftfahrt sowie Landwirtschaft, Verteidigung und andere Branchen der Energietechnik. Des Weiteren betreibt die FEV im Geschäftsbereich Test Systeme moderne Motoren-Prüfstandeinrichtungen und Messtechnik, die anfangs nur dem internen Gebrauch in Aachen dienten, seit Anfang der 90er Jahre jedoch auch für Kunden gefertigt werden. Das Angebot reicht von Testeinrichtungen bis hin zur Darstellung kundenindividueller, schlüsselfertiger Testzentren. Neben den genannten verfügt die FEV ebenfalls über Kompetenzen im Software-Engineering.

\subsection{Vorstellung der betrieblichen Teilprojekte}

\subsubsection{HELLA KGaA Hueck \& Co.: Qualifizierungskonzept für technologiebezogene Kompetenzen}

Die HELLA KGaA Hueck \& Co. sieht sich derzeit im Produktionsbereich mit zwei Trends konfrontiert. Einerseits ermöglichen die technologischen Entwicklungen der letzten Jahre eine zunehmende Automatisierung von Fertigungs- und Montageabläufen sowie der Produktionssteuerung. Dies hat einen Rückgang des Bedarfs an anzulernenden Montagekräften bei gleichzeitig steigendem Bedarf an qualifizierten technischen Fachkräften für hochautomatisierte Anlagen zur Folge. Andererseits steigt das Durchschnittsalter der Belegschaft in den deutschen Produktionsstandorten des Unternehmens stetig an. Dieser Umstand fordert das Unternehmen und die Beschäftigten gleichermaßen heraus, die sich 
stetig ändernden technischen und wirtschaftlichen Rahmenbedingungen und daraus resultierende Veränderungen bei den Tätigkeitsanforderungen zu meistern. Herkömmliche interne Qualifizierungsansätze ebenso wie externe Weiterbildungsangebote stoßen hier jedoch schnell an ihre Grenzen.

Die im Rahmen des Projektes ELIAS betrachteten Arbeitssysteme, in Abb. 7.4 schematisch dargestellt, sind bereits heute schon durch einen hohen Automatisierungsgrad bei einer hohen Produktkomplexität gekennzeichnet. Die Produktionsschritte laufen innerhalb verschiedener Teilmodule automatisiert ab. Die wenigen verbliebenen direkten manuellen Tätigkeiten beschränken sich bspw. auf das Einlegen sowie die Entnahme und Verpackung. Aufgrund der zunehmenden Anlagenkomplexität steigen die Anforderungen an das technische Personal deutlich an. Die Fachkraft Technik (FT) ist für die Betreuung der Produktionslinie zuständig und muss im Falle von Störungen regulierend eingreifen. Hierfür sind jedoch ein hohes Anlagenverständnis und Erfahrungswissen erforderlich. Bei komplexeren Störungen werden die Mitarbeiter des technischen Service informiert. Diese müssen über ein noch spezifischeres Systemverständnis verfügen und auf der Basis fundierten theoretischen Wissens sowie langjähriger praktischer Erfahrung in der Lage sein, die Produktionsanlage wieder instand zu setzen. Das erforderliche Wissen der Mitarbeiter des technischen Service wurde bisher im Rahmen von Schulungsmaßnahmen beim Betriebsmittellieferanten während der Inbetriebnahme einer neuen Produktionslinie vermittelt. Der Wissenstransfer auf weitere bzw. neue Mitarbeiter des technischen Service oder der Fachkräfte Technik erfolgte in Abhängigkeit des zur Verfügung stehenden erfahrenen Personals. Daher verfügen die Beschäftigten über variierende Qualifikationsniveaus und gehen bei der Bewältigung von Problemsituationen unterschiedlich vor, was ungeplante Stillstandzeiten der Produktionslinie zur Folge haben kann. Unterschiedliche Technologiestandards aufgrund verschiedener Anschaffungszeitpunkte, inkompatible Schnittstellen, aber auch nicht intuitiv auslesbare Störmeldungen an den Bedienpanels erschweren zudem das schnelle Einleiten adäquater Maßnahmen sowie einen flexiblen Personaleinsatz an mehreren unterschiedlichen automatischen Produktionslinien.

Um nun Betriebsmittel und Produktionsprozesse besser beherrschbar zu machen sowie im Problemfall die Arbeiten des technischen Service sinnvoll zu entlasten, besteht das Ziel der HELLA KGaA Hueck \& Co. darin, den erforderlichen Wissens- und Kompetenzaufbau der technischen Fachkräfte im Hinblick auf eine Förderung von Flexibilität, Systemkenntnis und -verständnis sowie Entscheidungskompetenz weiterzuentwickeln. Zunächst wurde eine detaillierte Analyse der Personalbedarfe, der Prozessabläufe sowie der Informationsbedarfe und -flüsse an den automatisierten Produktionslinien durchgeführt. Weiterhin wurde eine Technologiematrix erarbeitet, in der die werksweit eingesetzten Standardtechnologien erfasst sind. So konnte festgestellt werden, an welchen Produktionslinien mit vergleichbaren Technologien gearbeitet wird. Darauf aufbauend wurde ein Konzept für technologiebezogene Kompetenzen entwickelt. Dieses ordnet Tätigkeitsinhalte den entsprechenden Technologien zu und legt fest, welche Kompetenzen an welchen Produktionslinien erforderlich sind. Hiermit konnten Qualifikationsbedarfe aufgedeckt werden. Ein im Anschluss daran erarbeiteter Leitfaden für Unterweisungen ermöglicht 

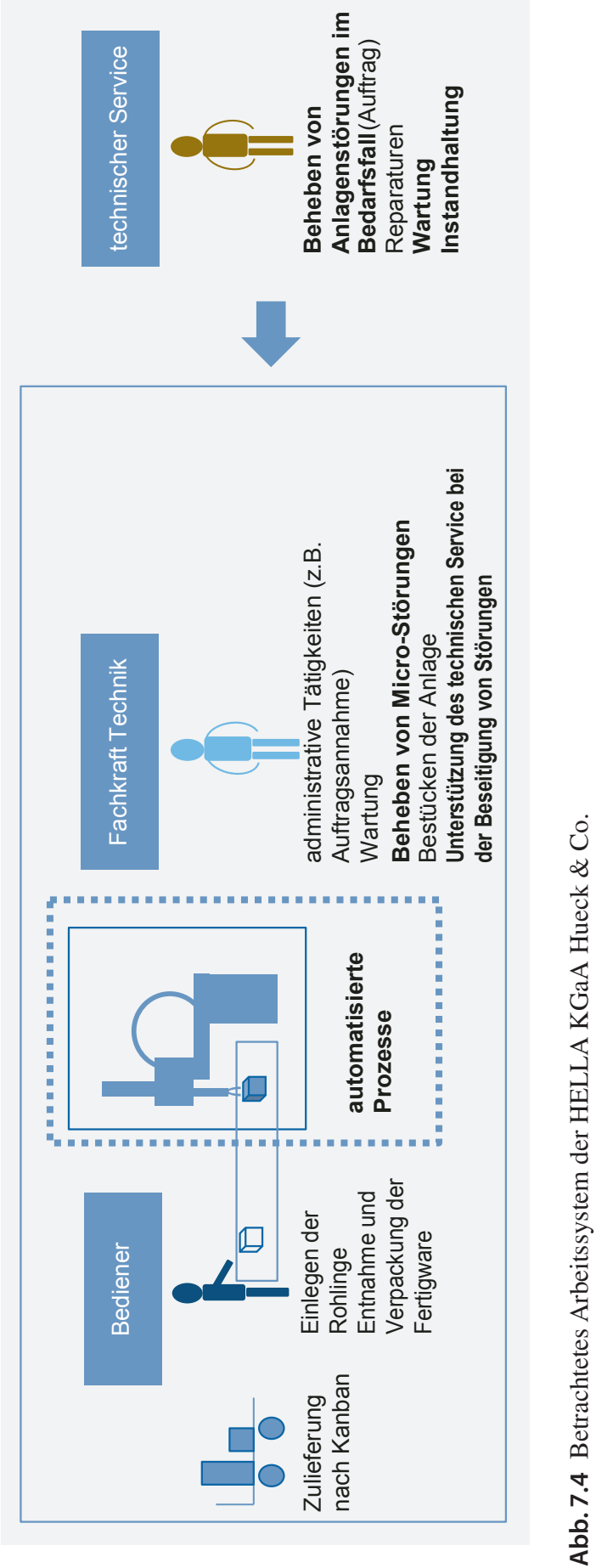
nun einerseits eine standardisierte Einarbeitung neuer Mitarbeiter und andererseits eine zielgerichtete Erhöhung bzw. Angleichung des Qualifikationsniveaus der Beschäftigten inklusive einer sukzessiven Erweiterung des Aufgabenspektrums der Fachkraft Technik.

\subsubsection{FEV GmbH: Modellbasierte Applikation von Steuergeräten}

Das Themenfeld, das im Rahmen des Projektes ELIAS in der FEV GmbH betrachtet wird, umfasst die Prozesse der Applikation elektronischer Steuergeräte an automobilen Antriebssträngen. Moderne Fahrzeuge sind mit einer Vielzahl an Steuergeräten ausgestattet, die z. B. für die Steuerung, Regelung und Überwachung von Motorfunktionen verantwortlich sind. Die Steuerungssoftware enthält eine Vielzahl von Einstellparametern (bis zu 30.000), die das Verhalten der Software beeinflussen. Ziel der Applikation ist es, Einstellparameter so zu definieren, dass bspw. Leistungswerte und Abgaswerte optimiert werden. Der Aufwand und der Zeitbedarf für die Applikation elektronischer Steuergeräte nehmen aufgrund strengerer Abgasgesetze sowie kontinuierlich komplexer werdender Systeme stetig zu. Zur Bestimmung der optimalen Einstellung der Steuergeräte müssen immer umfangreichere, im Voraus geplante Mess- und Einstellarbeiten am Prüfstand oder am Fahrzeug vorgenommen werden, bei denen der Applikationsingenieur (Applikateur) die verschiedenen Parameter des Steuergerätes an das Fahrzeug anpasst. Dabei müssen eine Vielzahl an Parametern und zahlreiche Wechselwirkungen beachtet werden. Aufgrund der immensen Komplexität sind für die Entwicklung, Kalibrierung und Diagnose von elektronischen Steuergeräten sowie zur Messdatenerfassung und -analyse softwaregestützte Applikationswerkzeuge unentbehrlich geworden. Die FEV hat daher Softwarelösungen zur modellbasierten Kalibrierung entwickelt. Die gerade beschriebene Situation ist in Abb. 7.5 visualisiert.

Modellbasierte Kalibrierungen ermöglichen die Planung der Versuche und deren automatische Durchführung am Prüfstand oder im Fahrzeug. Anhand der Messungen kann so das Fahrzeugverhalten modelliert werden. Außerdem können mittels weiterer

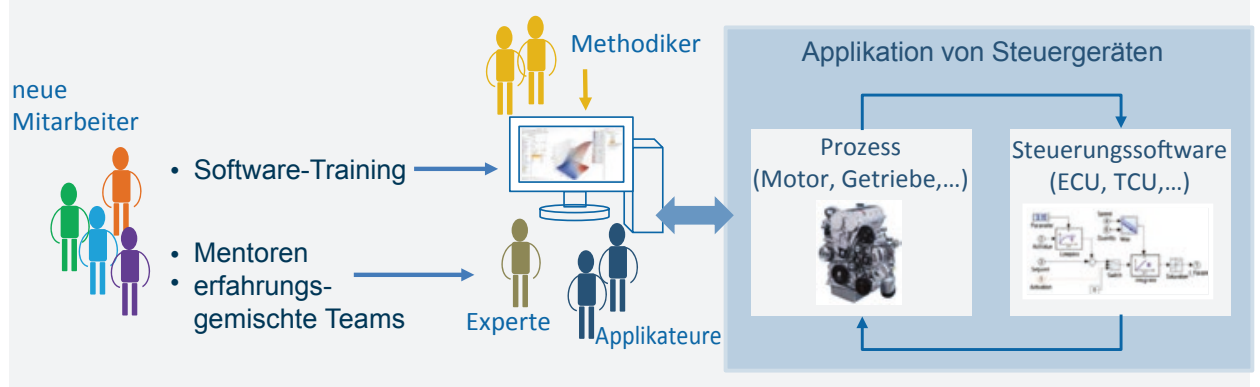

Abb. 7.5 Prozessablauf der modellbasierten Kalibrierung von Antriebssträngen bei der FEV 
Funktionalitäten die gemessenen Daten effizient analysiert sowie Datensätze optimiert werden. Mit diesen Softwarelösungen werden kostenintensive Motoren- und Fahrzeugversuche minimiert und Entwicklungszeiten verkürzt. Zudem wird die Qualität der Applikation gesteigert und Applikationsprozesse standardisiert.

Tätigkeiten im Bereich der Steuergeräte-Applikation erfordern heutzutage eine Einarbeitungs- und Ausbildungszeit von etwa 2 Jahren. Aufgrund der sich stetig weiterentwickelnden und an Komplexität zunehmenden Steuergerätefunktionen müssen sich jedoch die Applikateure kontinuierlich weiterbilden. Die unternehmensinterne Qualifizierung erfolgt zunächst über umfangreiche Schulungsmaßnahmen, die tätigkeitsspezifisch aufbereitet sind, da die unterschiedlichen Domänen wie Brennverfahrens-, Abgasnachbehandlungs- oder Fahrbarkeitsabstimmung organisatorisch überwiegend getrennt sind. Teil der Schulungsmaßnahmen ist ebenfalls die Vermittlung der Grundkenntnisse zum Umgang mit den entsprechenden Softwaretools zur modellbasierten Applikation, die zur Bearbeitung der Aufgaben und Projekte erforderlich sind. Die anschließende Einarbeitung in eine Domäne wird meist intuitiv von einfachen Teilaufgaben zu komplexen Gesamtsystemen strukturiert und durch die Arbeit in erfahrungsgemischten Teams unterstützt.

Auf die zunehmende Aufgabenkomplexität und -vielfalt sowie sich stetig verkürzende Entwicklungszyklen hat die FEV mit einer Ausweitung und Weiterentwicklung des Leistungs- bzw. Kompetenzspektrums und mit einem deutlichen Personalzuwachs reagiert. Das Unternehmen steht nun vor der Herausforderung, möglichst schnell einerseits die neuen Mitarbeiter für die Bewältigung der Projektaufgaben zu befähigen, sowie sich andererseits das notwendige Wissen in den neuen Aufgabengebieten anzueignen. Daher entwickelt die FEV GmbH im Rahmen des Projektes ein Konzept für ein lernförderlich gestaltetes, kognitives Assistenzsystem zur Applikation von Fahrzeugantrieben, um neue Mitarbeiter in diesen Aufgabenfeldern für die Datenanalyse und modellbasierte Kalibrierung schneller zu befähigen und langjährigen Mitarbeitern den Umgang mit der Software zu erleichtern. Die bisher verwendeten Softwaretools wurden in Ko-Evolution mit dem Aufgaben- und Kompetenzspektrum von erfahrenen Applikateuren selbst entwickelt und sukzessive an die steigenden Anforderungen angepasst. Da sie über keine integrierte Anleitung verfügten und wenig nutzerfreundlich waren, mussten die Applikateure, die mit diesen Tools arbeiteten, einerseits bereits über Systemverständnis verfügen, andererseits mit dem Tool vertraut sein, um die Projekte bewältigen zu können. Aufgabe der Methodiker ist es nun, in enger Zusammenarbeit mit Lead-Applikateuren, die über ein umfangreiches Erfahrungswissen verfügen, ein standardisiertes Softwaretool zu entwickeln, das unternehmensweit eingesetzt werden kann. Der Schwerpunkt der Arbeiten liegt dabei in einer visualisierten Darstellung der Prozesse sowie in einer Workflow-basierten Führung durch die Aufgabe. So soll den Applikateuren der Umgang mit der Software erleichtert und demzufolge die Einarbeitungszeit verkürzt werden. Da zudem repetitive Prozesse verstärkt automatisiert und standardisiert durchgeführt werden sollen, können diese Tätigkeiten zukünftig auch von weniger erfahrenen Mitarbeitern bewältigt werden. 


\subsection{Zu erwartende Auswirkungen der erarbeiteten bzw. durchgeführten Maßnahmen}

Im Folgenden wird dargestellt, welche Änderungen durch die Einführung der in Kap. 3 beschriebenen Konzepte bei den beiden Anwendungsfällen voraussichtlich zu erwarten sind. Zunächst erfolgen eine Beschreibung der betrieblichen Funktionen sowie eine Dokumentation der Maßnahmenauswirkungen auf diese Funktionen. Im folgenden Kapitel werden diese Änderungen zusammengefasst.

\subsubsection{Betroffene Tätigkeiten und Auswirkungen auf die betrieblichen Funktionen bei der HELLA KGaA Hueck \& Co.}

Die Bediener/Werker an den automatisierten Produktionsanlagen der HELLA KGaA Hueck \& Co. legen die Basisteile ein und verpacken die entnommene Fertigware. Neben administrativen Tätigkeiten ist die Fachkraft Technik (FT) vor allem für die Materialversorgung der Anlage und das Beheben von Mikro-Störungen zuständig. Das Aufgabengebiet der Mitarbeiter des technischen Service (TS) gliedert sich in die Reparatur, Wartung, Instandhaltung und vor allem die Beseitigung von Anlagenstörungen. Wegen der steigenden Anforderungen an die Fachkraft Technik werden zukünftig einige geeignete Fachkräfte Technik im Rahmen des erarbeiteten Qualifizierungskonzeptes zu High-LevelFachkräften Technik ausgebildet. Im Anschluss können diese die Arbeiten des technischen Service kompetent unterstützen bzw. Störungsbeseitigungs- und Instandsetzungsaufgaben in Teilen oder auch vollständig selbstständig als Spezialist durchführen. Da die Aufgaben der Teamleiter und der Meister nicht im Projektfokus liegen, findet eine weitere Betrachtung dieser Rollen hier nicht statt.

Grundsätzlich ist bei der HELLA KGaA Hueck \& Co. aufgrund der steigenden Anlagenund Produktkomplexität von einer steigenden Aufgabenkomplexität und damit einhergehenden Problemlösungsfähigkeiten bei den Fachkräften Technik, den Fachkräften Technik High Level sowie den Mitarbeitern des technischen Service auszugehen. Dies gilt im besonderen Maße für die Fachkräfte Technik High Level, da diese bei komplexeren Störungen von den verantwortlichen Fachkräften Technik hinzugezogen werden und eine Schnittstellenfunktion zwischen der operativen Fertigung und dem technischen Service übernehmen. Dagegen sind bei den Bedienern grundsätzlich keine Veränderungen zu erwarten. Einen Überblick über die Auswirkungen auf die betrieblichen Funktionen gibt Abb. 7.6.

Des Weiteren wird der Personalbedarf an Fachkräften Technik High Level deutlich steigen, die aber aus dem vorhandenen Personal von Fachkräften Technik rekrutiert werden. Der Anteil monotoner Tätigkeiten wird außer für die Fachkräfte Technik High Level, bei denen eine deutliche Reduktion dieser Arbeiten zu beobachten ist, gleichbleiben. Der Anteil planender und kontrollierender Tätigkeiten wird für die Fachkräfte Technik zunehmen, da häufiger zwei Linien betreut werden. Für die Fachkräfte Technik High Level werden beide Anteile ebenfalls ansteigen. 


\begin{tabular}{|c|c|c|c|c|}
\hline & Bediener & FT & FT high Level & TS \\
\hline Bedarf & 0 & - & ++ & 0 \\
\hline Problemlösung & 0 & + & ++ & + \\
\hline Monotone Aufgaben & o & 0 & -- & 0 \\
\hline Komplexe Aufgaben & o & + & ++ & + \\
\hline Planen & 0 & + & + & o \\
\hline Kontrolle & 0 & 0 & + & o \\
\hline Lernen, informell & 0 & + & ++ & + \\
\hline Lernen, formell & o & + & ++ & o \\
\hline Selbstbestimmung & o & o & + & o \\
\hline Optimierung & o & + & ++ & 0 \\
\hline Kooperation & o & + & ++ & + \\
\hline Kommunikation & 0 & + & ++ & + \\
\hline Interdisziplinarität & 0 & 0 & 0 & 0 \\
\hline ITKenntnisse & 0 & + & ++ & + \\
\hline $\begin{array}{l}\text { Legende: } \\
-\quad \text { wird viel weniger }\end{array}$ & wird weniger & bleibt gleich & wird mehr & wirc \\
\hline
\end{tabular}

Abb. 7.6 Auswirkungen auf die betrieblichen Funktionen der HELLA KGaA Hueck \& Co.

Das arbeitsnahe Lernen sowohl in informeller als auch formeller Form wird deutlich zunehmen. Hierbei wird auch das im Rahmen des Verbundprojektes ELIAS entwickelte Assistenzsystem der HELLA KGaA Hueck \& Co. eine zentrale Rolle spielen. Während die Fachkräfte Technik High Level mit einem deutlichen Anstieg an Lernprozessen konfrontiert sind, wird die verstärkte Kommunikation und Kooperation zwischen den drei Rollen auch das informelle Lernen positiv beeinflussen. Dabei ist im besonderen Maße die Schnittstellenfunktion zum technischen Service der Fachkräfte Technik High Level hervorzuheben. Gleichzeitig übernehmen die Fachkräfte Technik High Level eine Schlüsselfunktion bei der Qualifizierung der technischen Fachkräfte, da erworbenes Wissen aus der Zusammenarbeit mit dem technischen Service weitergegeben werden kann. In Bezug auf die Selbstbestimmung sind kaum Veränderungen zu erwarten, wobei eine Erweiterung der Befugnisse für die Fachkräfte Technik High Level zu erwarten ist. Die Optimierung betreffend ist für alle Rollen eine Zunahme zu erwarten, da insbesondere durch den im Assistenzsystem geplanten Feedbackloop eine kontinuierliche Verbesserung der Maßnahmen zu Störungsbehebung erreicht werden soll. Die Anforderungen hinsichtlich interdisziplinären Wissens, das über die Kenntnisse im eigenen Fachbereich hinausgeht, bleiben für den technischen Service unverändert, steigen aber für die Fachkräfte Technik und besonders für die Fachkräfte Technik High Level, da sowohl mechanische als auch elektronische Mikrostörungen zukünftig selbstständig gelöst werden. Die zunehmende Informatisierung der Instandhaltung sowie die komplexer werdende Steuerung neuer Anlagen erhöhen auch die Anforderungen an die IT-Kompetenzen aller 
Beteiligten, wobei die deutlichste Zunahme wiederum für die Fachkräfte Technik High Level zu erwarten ist.

\subsubsection{Betroffene Tätigkeiten und Auswirkungen auf die betrieblichen Funktionen bei der FEV GmbH}

Die Applikationsingenieure (Applikateure) der FEV GmbH führen die Kalibrierung der Steuergeräte durch und bearbeiten dabei unterschiedliche Aufgaben oder Projekte. LeadApplikateure sind langjährige Mitarbeiter mit einem umfangreichen Erfahrungswissen. Die Teilprojektleiter sind verantwortlich für operative Planung und Steuerung im spezialisierten Arbeitsbereich. Der Gesamtprojektleiter trägt die Verantwortung für das operative Planen und Steuern des Projektes sowie für das Erreichen aller Projektziele. Die Methodiker erarbeiten strukturiert und eigenständig Methoden in den verschiedensten Bereichen, z. B. des Software-Engineerings, und sind für die Koordination und Durchführung von Schulungen zuständig. Aufgrund der umfangreichen Erweiterung des Leistungsangebots wird zukünftig das Tätigkeitsspektrum der Applikateure weiter ausdifferenziert. Es werden aber keine weiteren betrieblichen Funktionen hinzukommen.

Wie sich die betrieblichen Funktionen der FEV GmbH in Zukunft verändern werden, ist in Abb. 7.7 dargestellt. Der Bedarf an Personal wird in allen fünf Funktionen der FEV $\mathrm{GmbH}$ deutlich steigen, was auf das starke Wachstum der FEV GmbH zurückzuführen ist. Lediglich in Domänen, die seit vielen Jahren im Unternehmen etabliert sind, bleibt die Anzahl an Lead-Applikateuren in etwa gleich. Für Domänen, die hinzukommen werden, besteht Bedarf an neuen Lead-Applikateuren und Methodikern. Da die Anzahl

\begin{tabular}{|c|c|c|c|c|c|}
\hline & Methodiker & Applikateur & $\begin{array}{l}\text { Lead-Applikateur } \\
\text { (Experte) }\end{array}$ & Teilprojektleiter & Gesamtprojektleiter \\
\hline Bedarf & + & ++ & + & ++ & ++ \\
\hline Problemlösung & ++ & - & + & 0 & 0 \\
\hline Monotone Aufgaben & k.A. & k.A. & k.A. & k.A. & k.A. \\
\hline Komplexe Aufgaben & + & + & ++ & + & + \\
\hline Planen & 0 & 0 & 0 & ++ & ++ \\
\hline Kontrolle & 0 & - & + & + & + \\
\hline Lernen, informell & 0 & + & + & 0 & 0 \\
\hline Lernen, formell & ++ & + & ++ & + & + \\
\hline Selbstbestimmung & + & 0 & + & 0 & 0 \\
\hline Optimierung & + & 0 & + & ++ & + \\
\hline Kooperation & + & 0 & + & 0 & ++ \\
\hline Kommunikation & + & + & + & + & ++ \\
\hline Interdisziplinarität & ++ & 0 & + & 0 & + \\
\hline IT-Kenntnisse & 0 & + & 0 & 0 & o \\
\hline $\begin{array}{l}\text { Legende: } \\
--\quad \text { wird viel weniger }\end{array}$ & wird we & o & t gleich & wird mehr & $++\quad$ wird viel mehr \\
\hline
\end{tabular}

Abb. 7.7 Auswirkungen auf die betrieblichen Funktionen der FEV GmbH 
der Projekte stark zunehmen wird, werden zukünftig deutlich mehr Applikateure, Teilund Gesamtprojektleiter benötigt.

Die Anforderungen eigenständiger Lösungen für nicht vorhersagbare Probleme werden insbesondere bei den Methodikern, aber auch bei den Lead-Applikateuren aufgrund der zunehmenden Anzahl an Domänen und Aufgaben steigen. Da es kaum monotone Aufgaben gab und zukünftig auch nicht geben wird, konnten hier keine weiteren Angaben gemacht werden. Der Anteil komplexer Arbeitsinhalte wird bei allen Funktionen steigen. Besonders betroffen sind die Lead-Applikateure, da sie häufig neue Aufgaben bearbeiten, bei denen das notwendige Wissen noch nicht vorhanden ist. Für Teil- und Gesamtprojektleiter wird der Anteil an planerischen Aktivitäten aufgrund der steigenden Anzahl an Projekten und Aufgaben stark zunehmen. Der Anteil kontrollierender und überwachender Tätigkeiten wird sich für Methodiker nicht ändern, für Applikateure aufgrund zunehmender Standards abnehmen, für Lead-Applikateure, Teil- und Gesamtprojektleiter jedoch wegen der steigenden Aufgabenkomplexität erhöhen.

Die Notwendigkeit des informellen Lernens im Prozess der Arbeit besteht nach wie vor für alle Funktionen, wird aber wegen der steigenden Aufgabenkomplexität für Applikateure und Lead-Applikateure zunehmen. Die Notwendigkeit des formellen Lernens nimmt für alle Funktionen zu, besonders aber für Methodiker und Lead-Applikateure (Bewältigung neuer komplexer Aufgaben). Der Grad der Selbstbestimmung bleibt für die meisten Funktionen gleich, wird aber für Methodiker und Lead-Applikateure steigen, die durch individuelles Handeln und das Einbringen von eigenen Ideen die Weiterentwicklung von Standards und Methoden unterstützen können. Methodiker, Lead-Applikateure und Gesamtprojektleiter werden zukünftig verstärkt die Möglichkeit haben, ihre eigene Arbeit zu optimieren. Aufgrund vieler neuer Aufgaben, bei denen die Vorgehensweise noch unbekannt ist, sind aber insbesondere die Teilprojektleiter gefordert, optimale Lösungen zu finden. Für Methodiker, Lead-Applikateure und besonders für Gesamtprojektleiter werden die Anforderungen an ihre kooperativen Fähigkeiten steigen, da ihre Tätigkeiten verstärkt in internationale Projektstrukturen eingebunden werden (horizontale Vernetzung im Unternehmen) und dabei ein erhöhter Abstimmungsbedarf besteht. Für alle Funktionen, insbesondere für Gesamtprojektleiter, wird es wichtiger werden, ihre kommunikativen Fähigkeiten zu stärken. Interdisziplinäres Wissen, das über die Kenntnisse im eigenen Fachbereich hinausgeht, wird besonders relevant für Methodiker, aber auch für Lead-Applikateure und Gesamtprojektleiter. Da IT-Kompetenz zur Bewältigung der alltäglichen Arbeit bisher schon zwingend erforderlich war, werden sich die Anforderungen an die IT-Kenntnisse nur für Applikateure erhöhen, da ihre Aufgaben verstärkt mittels Unterstützung durch Softwaretools ausgeführt werden.

\subsection{Zusammenfassende Betrachtung der Auswirkungen}

Die im Vorangegangenen beschriebenen Änderungen werden an dieser Stelle detailliert zusammengefasst. Betrachtet wird dabei, wie sich die eingeführten Maßnahmen auf die Arbeitsaufgaben und -tätigkeiten der betroffenen betrieblichen Funktionen auswirken. 
Veränderungen bezüglich der Arbeitsorganisation, des Arbeitsplatzes und der -umgebung werden dabei ebenso erfasst wie die zukünftig eingesetzten Arbeitsmittel. Von Bedeutung wird ebenfalls sein, welche Effekte bei der Qualifikations- und Kompetenzentwicklung erzielt werden und ob es im Bereich der Führung zu Veränderungen kommen wird.

\subsubsection{Auswirkungen bei der HELLA KGaA Hueck \& Co.}

Während für Bediener/Werker und den technischen Service bei der HELLA KGaA Hueck \& Co. kaum Veränderungen zu erwarten sind, sehen sich die Fachkräfte Technik sowie Fachkräfte Technik High Level einem deutlichen Wandel gegenüber. Einen Überblick der Auswirkungen bei der HELLA KGaA Hueck \& Co. gibt Abb. 7.8.

Da die Fachkraft Technik High Level den technischen Service zunehmend in seiner Arbeit unterstützt, werden ihre Arbeitsaufgaben erweitert und mit neuen Inhalten angereichert. Sie hat ein breiteres Spektrum an zudem komplexeren Aufgaben zu bewältigen. Die Fachkraft Technik wird mit ähnlich komplexen Aufgaben wie zuvor betraut sein. Zusätzlich zum bekannten Arbeitsplatz kann die Fachkraft Technik zukünftig an weiteren Produktionslinien eingesetzt werden. Die Fachkraft Technik High Level wird nach Erlangung der erforderlichen Qualifikationen an verschiedenen Linien eingesetzt. Zur Verrichtung ihrer täglichen Arbeiten werden den Fachkräften Technik und den Fachkräften Technik High Level zusätzliche Arbeitsmittel in Form eines technologiegestützten Assistenzsystems zur Verfügung gestellt. Im Fokus des geplanten Assistenzsystems stehen dabei Anleitungen zur Fehlerbehebung, die iterativ von den Fachkräften Technik mit Unterstützung des technischen Service entwickelt werden. Hierbei ist ein integrierter Zugang zu Lernmaterialien sowie zu digitalisierten Maschinenbögen denkbar. Für die Mehrlinienbedienung findet zudem derzeit eine simulationsbasierte Optimierung der Wege innerhalb des Arbeitssystems statt, die im späteren Verlauf auch zur Planungsunterstützung eingesetzt werden kann. Gleichzeitig ist von einer weiteren Zunahme der Komplexität der Produktionsanlagen auszugehen. Bei der Arbeitsorganisation wird es zu einer Änderung der Personalstruktur kommen. Die Anzahl der Fachkräfte Technik High Level wird sich erhöhen, die der Fachkraft Technik damit relativ gesehen sinken. Personalbedingte Stillstandzeiten können durch einen effizienteren und flexibleren Einsatz der Fachkräfte Technik an mehreren Linien reduziert werden. Außerdem ist ein verstärkter Austausch zwischen allen Rollen zu erwarten. Für die Fachkräfte Technik High Level wird es vermehrt zu Jobrotation kommen; außerdem werden Arbeits- und Lernaufgaben gemeinsam mit dem technischen Service ausgeführt. Neben der neu geschaffenen Rolle der Fachkraft Technik High Level wird insbesondere die vermehrte technologiegestützte Informationsbereitstellung für alle Fachkräfte Technik zu Veränderungen in der Arbeitsumgebung führen. Aufgrund der zunehmenden Komplexität der Produktionsanlagen sowie der verstärkten Verlagerung von Aufgaben wie der Behebung von Mikrostörungen ist ein Qualifikationsbedarf für alle Fachkräfte Technik und im besonderen Maße für die Fachkräfte Technik High Level 


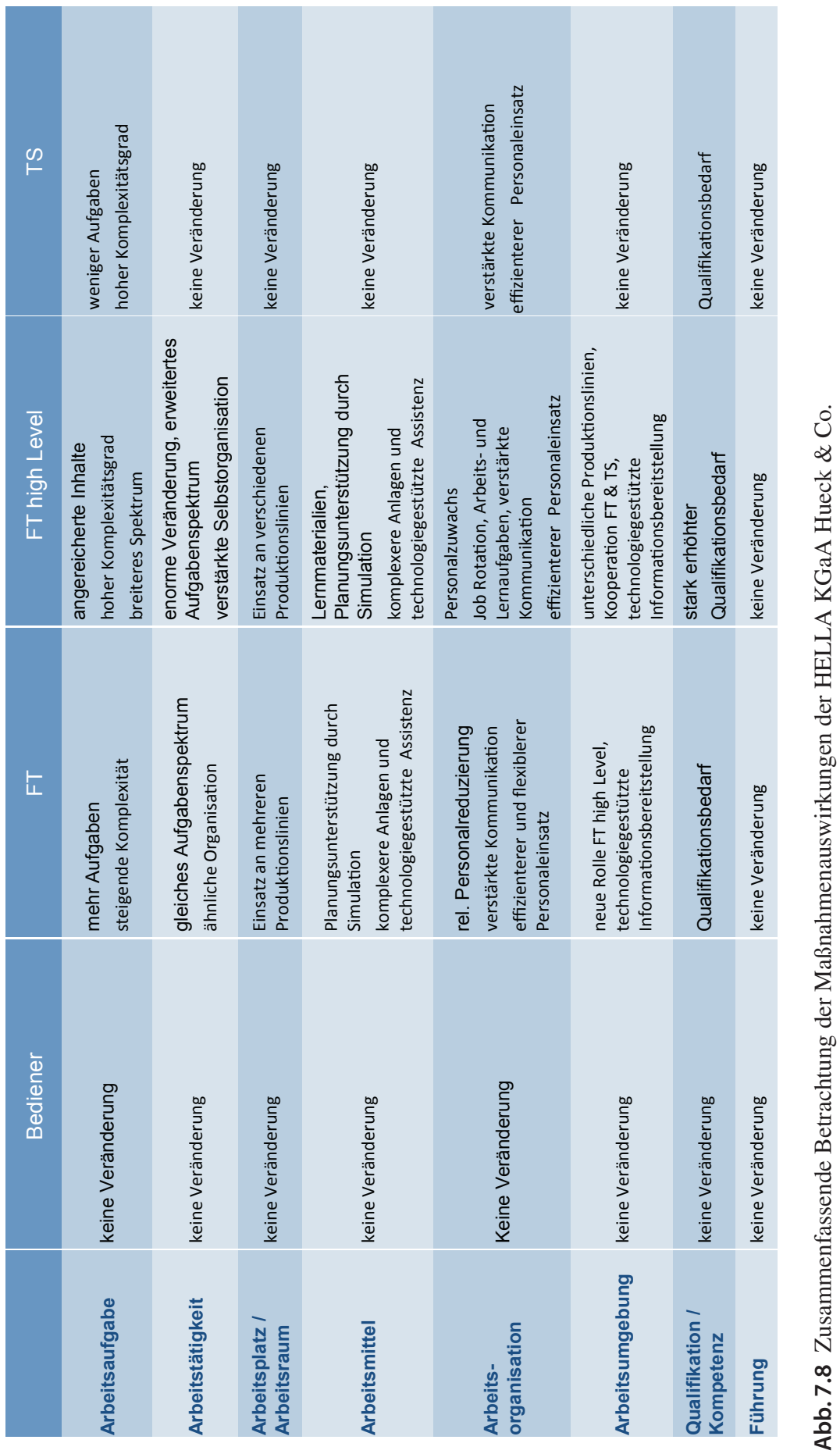


zu konstatieren. Zusätzliche leitende oder führende Tätigkeiten werden von keiner der betrachteten betrieblichen Funktionen ausgeübt.

\subsubsection{Auswirkungen bei der FEV GmbH}

Fast alle betroffenen betrieblichen Funktionen in der FEV GmbH sind mit zunehmend komplexer werdenden Aufgaben konfrontiert. Zudem werden aufgrund des sich erweiternden Leistungsangebotes neue Aufgaben auf die Methodiker und Lead-Applikateure zukommen. Teil- und Gesamtprojektleiter sind zunehmend in die Koordination internationaler Projekte eingebunden. In Abb. 7.9 werden die Auswirkungen der eingeleiteten Maßnahmen bei der FEV GmbH gezeigt.

Während bei den meisten Funktionen eine Erweiterung des Tätigkeitsspektrums prognostiziert werden kann, ist bei dem Tätigkeitsspektrum der Applikateure eine weitere Ausdifferenzierung der Tätigkeit aufgrund der steigenden Komplexität durch vermehrt parallel zu betreibende Systeme zu erwarten. Neue Softwaretools mit integrierten Lerninhalten werden den Applikateuren, Lead-Applikateuren und Teilprojektleitern als unterstützende Arbeitsmittel zur Verfügung gestellt. Bei der Arbeitsorganisation wird es zu einer Veränderung der Personalstruktur kommen. Für eine Abdeckung des angebotenen Leistungsspektrums ist eine deutlich höhere Anzahl an Applikateuren erforderlich, deren Tätigkeitsschwerpunkte dementsprechend ausdifferenziert werden. Langfristig ist geplant, den Aufgabenanteil der Applikateure, der am Fahrzeug ausgeführt werden muss, zu verringern und diesen verstärkt simulationsbasiert auszuführen. Durch die steigenden Anforderungen und den erhöhten Komplexitätsgrad ergeben sich steigende Qualifikationsbedarfe für die Applikateure und Lead-Applikateure. Für Methodiker und Teilprojektleiter gewinnt das informelle Lernen immer mehr an Bedeutung. Zudem wird aufgrund der stark zunehmenden Zahl internationaler Projekte die interkulturelle Kompetenz von Teilprojektleitern gefordert sein. Eine Erweiterung der Führungsaufgaben ist für keine der betrachteten Positionen vorgesehen.

\subsection{Fazit}

Die beiden dargestellten Cases unterscheiden sich in vielfältiger Hinsicht, sodass eine direkte Vergleichbarkeit zunächst kaum gegeben zu sein scheint. So sind Produkte, Technologien und Herausforderungen verschieden. Bei aller Unterschiedlichkeit ist ihnen aber gemein, dass sie betriebliche Reaktionen auf einen Komplexitätsanstieg sind. Im Fall HELLA handelt es sich um eine längerfristige, in kleinen bis größeren Sprüngen und an verschiedenen Produktionslinien unterschiedlich schnell stattfindende Zunahme der Anlagenkomplexität. Zwar schlägt sich diese nicht in höheren Anforderungen an die Bedienung im Normalbetrieb nieder, wohl aber in steigenden Anforderungen hinsichtlich der Minimierung von Anlagenstillständen und der Maximierung der Anlageneffektivität. Im 


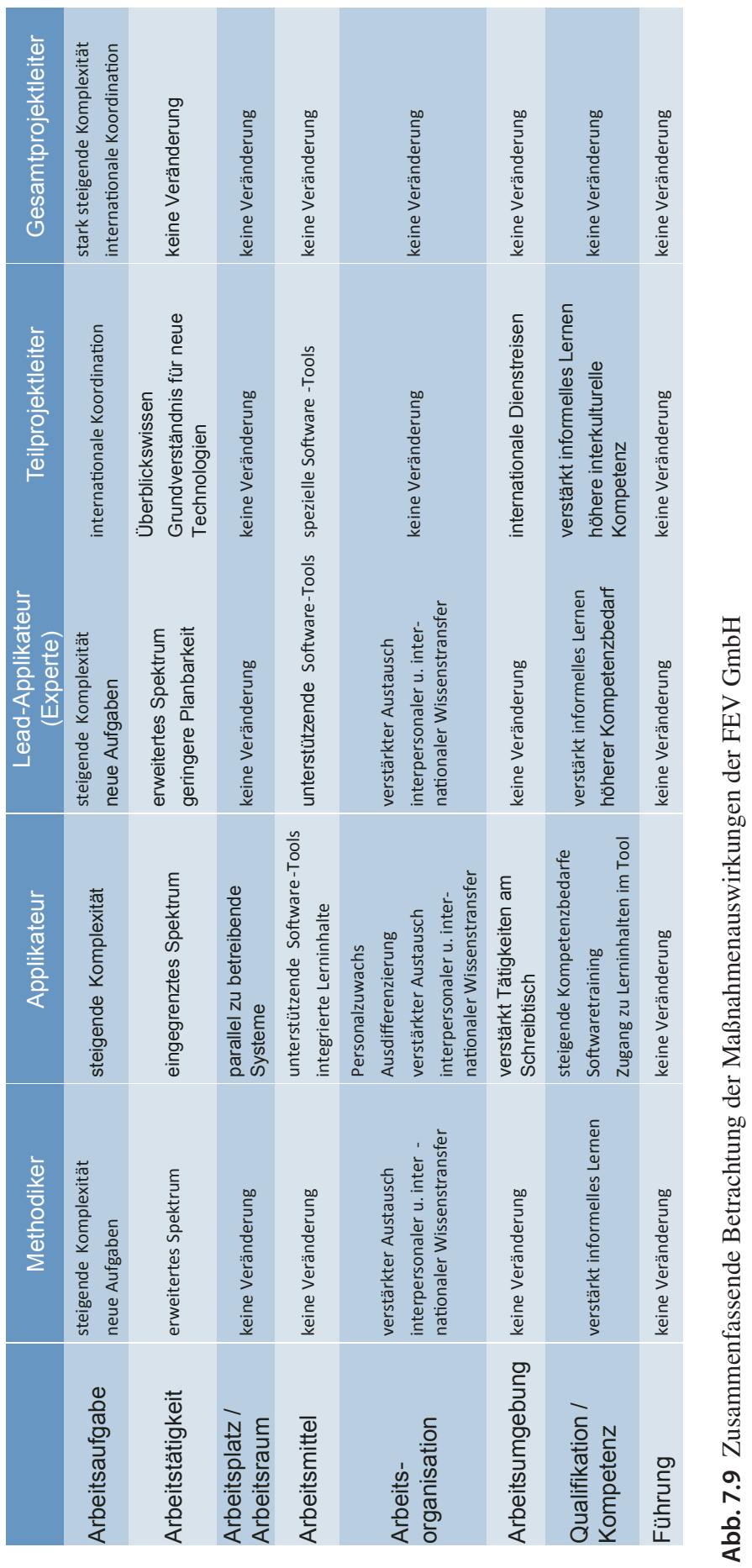


Fall FEV handelt es sich um die längerfristige Zunahme der Produktkomplexität, indem die Dienstleistungen variantenreicher werden und die Auftragskomplexität pro Auftrag ebenfalls zunimmt.

Vor diesem gemeinsamen Hintergrund lassen sich auch in den gewählten Vorgehensweisen und Lösungsansätzen grundlegende Übereinstimmungen zwischen beiden Fällen aufzeigen:

- Es kommen digitale Werkzeuge zum Einsatz, die der Assistenz bei der Aufgabendurchführung dienen und sowohl das individuelle als auch das organisationale Lernen adressieren.

- Es finden Veränderungen der Arbeitsorganisation statt, indem Tätigkeitsinhalte verlagert werden und neue Stellenprofile entstehen.

- Es finden Qualifizierungsprozesse statt und neue Personaleinsatz- und -entwicklungskonzepte entstehen.

Mensch, Technik und Organisation (MTO) bilden die tragenden und miteinander verbundenen Elemente der Lösung beider Cases. Aufgrund dieser Übereinstimmung lassen sich auch gemeinsame Muster in der Vorgehensweise in beiden Unternehmen aufzeigen. Die Entwicklung digitaler Werkzeuge wird im Unternehmen selbst vorgenommen unter Einbeziehung des Sachverstandes verschiedener Akteure und in einem Prototyping-Verfahren, welches auch die Nutzer in die Entwicklung und Erprobung einbezieht. So kann gefolgert werden, dass sich angemessene digitale Werkzeuge nicht als fertige Lösungen „Off-theShelf“ einkaufen lassen. Stattdessen werden diese intern, mit starkem Bezug zu Tätigkeitsinhalten und betrieblichem Wissen, entwickelt. Entsprechend sind einschlägige Kompetenzen zu Konzeption, Arbeitsanalyse und Beteiligungsmanagement erforderlich. Diese müssen teilweise aufgrund fehlender Vorerfahrungen, im Prozess selbst erworben werden.

Des Weiteren findet der Aufbau von Kompetenzen sowie das organisationale Lernen in Form arbeitsnaher und arbeitsintegrierter Ansätze (Mühlbradt et al. 2015; Senderek et al. 2015; Mühlbradt 2014) statt. Dies betrifft das individuelle Lernen zum Aufbau von Kompetenzen, beispielsweise durch Coaching und Job-Rotation, aber auch das organisationale Lernen durch die Integration entsprechender Funktionalitäten in die Digitalen Werkzeuge und in Arbeitsprozesse. Nicht zuletzt zeigen beide Fälle, dass die Informatisierung (Botthof 2015, S. 4) und Automatisierung der Arbeitswelt nicht gleichbedeutend mit dem Wegfall von geringqualifizierter Arbeit sein muss.

\section{Literatur}

BITKOM. (2014). Digitalisierung schafft rund 1,5 Millionen Arbeitsplätze (Pressemitteilung). https://www.bitkom.org/Presse/Presseinformation/Pressemitteilung_4608.html. Zugegriffen: 01. Dez. 2015.

BITKOM und Fraunhofer IAO. (2014). Industrie 4.0 Volkswirtschaftsliches Potenzial für Deutschland. Berlin: Bundesverband Informationswirtschaft, Telekommunikation und neue Medien e. V. Botthof, A. (2015). Zukunft der Arbeit im Kontext von Autonomik und Industrie 4.0. In A. Botthof \& E. A. Hartmann (Hrsg.), Zukunft der Arbeit in Industrie 4.0 (S. 4-6). Berlin: Springer Vieweg. 
Frey, C. B., \& Osborne, M. A. (2013). The future of employment: How susceptible are jobs to computerisation? http://www.google.de/url?sa=t\&rct=j\&q=\&esrc=s\&source=web\&cd=1\&cad=rja\&uact= $8 \& v e d=0 \mathrm{CCYQFjAA} \& u r l=\mathrm{http} \% 3 \mathrm{~A} \% 2 \mathrm{~F} \% 2 \mathrm{Fwww}$. oxfordmartin.ox.ac.uk $\% 2 \mathrm{Fdownloads} \% 2 \mathrm{Fa}-$ cademic\%2FThe_Future_of_Employment.pdf\&ei=oeobVePSOobXUbqeg5gJ\&usg=AFQjCNFu jcxMopCcadhq7bdkRjZoy0qCYg\&bvm=bv.89744112,d.bGg. Zugegriffen: 01. Apr. 2015.

Kagermann, H., Wahlster, W., \& Helbig, J. (2013). Umsetzungsempfehlungen für das Zukunftsprojekt Industrie 4.0 - Deutschlands Zukunft als Produktionsstandort sichern. Berlin: Promotorengruppe Kommunikation der Forschungsunion Wirtschaft - Wissenschaft.

Kelkar, O., Heger, R., \& Dao, D.-K. (2014). Industrie 4.0 - Eine Standortbestimmung der Automobilund Fertigungsindustrie - Zusammenfassung. http://www.mhp.com/de/home/. Online unter: http://www.mhp.com/fileadmin/mhp.de/assets/studien/MHP-Studie_Industrie4.0_V1.0.pdf. Zugegriffen: 04. Nov. 2015.

Lukas, U. v., Stork, A., \& Behr, J. (2014). Industrie 4.0 - Evolution statt Revolution. Visual Computing beflügelt die Industrie der Zukunft. wt Werkstattstechnik online, 104(4), 255-257.

Mühlbradt, T. (2014). Was macht Arbeit lernförderlich? - Eine Bestandsaufnahme. In MTM-Schriften Industrial Engineering. Zeuthen: Hrsg.: Deutsche MTM-Vereinigung e. V.

Mühlbradt, T., Senderek, R., Rodenhauser, T., \& Saupp, L. (2015). Arbeitsorientierte Lernlösungen für Industrielle Arbeitssysteme: Lernen für die Arbeit. In MTM-Schriften Industrial Engineering. Ausgabe 2. Zeuthen: Hrsg.: Deutsche MTM-Vereinigung e. V.

Müller, K. (2015). Fachkräfte für die intelligente Produktion - Aus- und Weiterbildungskonzepte für Industrie 4.0. Thementag 2015: „Industrie 4.0 - Handlungsfelder und Herausforderungen“. http://vhs4business.de/fileadmin/PDF_Seminare/Thementag_Beitraege/Karlheinz_Mueller_VDMA.pdf.

Pierre Audoin Consultants GmbH. (2014). IT Innovation Readiness Index 2014. Weinheim: Freudenberg IT SE \& Co. KG.

Rüßmann, M., Lorenz, M., Gerbert, P., Waldner, M., Justus, J., Engel, P., \& Harnisch, M. (2015). Industry 4.0. The future of productivity and growth in manufacturing industries.https://www. bcgperspectives.com/Images/Industry_40_Future_of_Productivity_April_2015_tcm80185183.pdf.Zugegriffen: 22. Apr. 2015.

Senderek, R., Mühlbradt, T., \& Buschmeyer, A. (2015). Demografiesensibles Kompetenzmanagement für die Industrie 4.0. In Exploring Demographics (S. 281-295). Springer.

Spath, D., Ganschar, O., Gerlach, S., Hämmerle, M., Krause, T., \& Schlund, S. (2013). In D. Spath (Hrsg.), Produktionsarbeit der Zukunft - Industrie 4.0. Stuttgart: Fraunhofer Verlag.

Tutt, C. (2015). Abschied von der Arbeit. Wirtschaftswoche, 5(2014), 18-23.

Open Access Dieses Kapitel wird unter der Creative Commons Namensnennung 4.0 International Lizenz (http://creativecommons.org/licenses/by/4.0/deed.de) veröffentlicht, welche die Nutzung, Vervielfältigung, Bearbeitung, Verbreitung und Wiedergabe in jeglichem Medium und Format erlaubt, sofern Sie den/die ursprünglichen Autor(en) und die Quelle ordnungsge-mäß nennen, einen Link zur Creative Commons Lizenz beifügen und angeben, ob Änderungen vorgenommen wurden.

Die in diesem Kapitel enthaltenen Bilder und sonstiges Drittmaterial unterliegen ebenfalls der genannten Creative Commons Lizenz, sofern sich aus der Abbildungslegende nichts anderes ergibt. Sofern das betreffende Material nicht unter der genannten Creative Commons Lizenz steht und die betreffende Handlung nicht nach gesetzlichen Vorschriften erlaubt ist, ist für die oben aufgeführten Weiterverwendungen des Materials die Einwilligung des jeweiligen Recht-einhabers einzuholen.

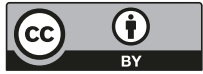

